\title{
Ti adatom diffusion on TiN(001): Ab initio and classical molecular dynamics simulations
}

\author{
Davide Sangiovanni, Daniel Edström, Lars Hultman, Ivan Petrov, Joseph E Greene and \\ Valeriu Chirita
}

\section{Linköping University Post Print}

\section{Tweet}

N.B.: When citing this work, cite the original article.

Original Publication:

Davide Sangiovanni, Daniel Edström, Lars Hultman, Ivan Petrov, Joseph E Greene and Valeriu Chirita, Ti adatom diffusion on $\mathrm{TiN}(001)$ : $\mathrm{Ab}$ initio and classical molecular dynamics simulations, 2014, Surface Science, (627), 34-41.

http://dx.doi.org/10.1016/j.susc.2014.04.007

Copyright: Elsevier

http://www.elsevier.com/

Postprint available at: Linköping University Electronic Press

http://urn.kb.se/resolve?urn=urn:nbn:se:liu:diva-109215 


\title{
Ti adatom diffusion on TiN(001): $a b$ initio and classical molecular dynamics simulations
}

\author{
D.G. Sangiovanni, ${ }^{a^{*}}$ D. Edström, ${ }^{a}$ L. Hultman, ${ }^{a}$ I. Petrov,,${ }^{a, b}$ J.E. Greene, ${ }^{a, b}$ V. Chirita ${ }^{a}$ \\ aDepartment of Physics, Chemistry and Biology (IFM) \\ Linköping University, SE-58183 Linköping, Sweden \\ ${ }^{b}$ Departments of Materials Science, Physics, and the Frederick Seitz Materials Research \\ Laboratory, University of Illinois, Urbana, IL 61801, USA
}

\begin{abstract}
$A b$ initio and classical molecular dynamics (AIMD and CMD) simulations reveal that Ti adatoms on TiN(001) surfaces migrate between neighboring fourfold hollow sites primarily along in-plane $<100>$ channels. $<100>$ and $<110>$ single jumps, as well as $<100>$ double jump rates, obtained directly from MD runs as a function of temperature, are used to determine diffusion activation energies $\mathrm{E}_{\mathrm{a}}$, and attempt frequencies $\mathrm{A}$, for the three preferred Ti adatom migration pathways on $\operatorname{TiN}(001)$. From transition rates $A \exp \left[-\mathrm{E}_{\mathrm{a}} /\left(\mathrm{k}_{\mathrm{B}} \mathrm{T}\right)\right]$, we determine adatom surface distribution probabilities as a function of time, which are used to calculate adatom diffusion coefficients $\mathrm{D}_{\mathrm{s}}(\mathrm{T})$. AIMD and CMD predictions are consistent and complementary. Using CMD, we investigate the effect on the adatom jump rate of varying the phonon wavelength degrees of freedom by progressively increasing the supercell size. We find that long-wavelength phonons significantly contribute to increasing adatom mobilities at temperatures $\leq 600 \mathrm{~K}$, but not at higher temperatures. Finally, by directly tracking the Ti adatom mean-square displacement during CMD runs, we find that $\mathrm{Ti}$ adatom jumps are highly correlated on $\mathrm{TiN}(001)$, an effect that yields lower $\mathrm{D}_{\mathrm{s}}$ values $\left(D_{s}^{\text {corr }}\right)$ than those estimated from uncorrelated transition probabilities. The temperature-dependent diffusion coefficient is $\mathrm{D}_{\mathrm{s}}^{\mathrm{cor}}(\mathrm{T})=\left(4.5 \times 10^{-4} \mathrm{~cm}^{2} \mathrm{~s}^{-1}\right) \exp \left[-0.55 \mathrm{eV} /\left(\mathrm{k}_{\mathrm{B}} \mathrm{T}\right)\right]$.
\end{abstract}

Keywords: Surface diffusion; Nitrides; Molecular dynamics simulations; Density functional theory *Corresponding author: Davide G. Sangiovanni, Tel. 0046 13282623, Fax 004613137568 , e-mail : davsan@ifm.liu.se 


\section{Introduction}

Transition-metal (TM) nitrides exhibit excellent physical and mechanical properties, including high hardness [1,2] and toughness [3], chemical inertness, and good thermal stability and electrical conductivity [4]. Thus, they are employed in a wide variety of applications ranging from wear-resistant protective coatings on cutting tools and engine components $[5,6]$ to diffusion barriers in electronic devices [7-11]. The properties and performance of TM nitrides layers ultimately depend on their nanostructure. During thin-film deposition, nanostructural and surface morphological evolution are largely controlled by the dynamics of adatom surface migration which, in turn, determine nucleation kinetics and film growth modes. A deeper understanding of these processes may allow better control of growth dynamics, and thus the ability to tailor film properties to specific requirements.

Very little is known about either the mechanisms or the kinetics of mass transport in TM nitride systems [12-14]. For TiN, the most studied and best characterized TM nitride [15], differences in reported bulk diffusion coefficients range over several orders of magnitude $[14,16,17]$. Moreover, surface mass transport properties lack experimental verification. This is due to the fact that even advanced atomic-scale experimental techniques, such as scanning tunneling microscopy [18-20] and low-energy electron microscopy [21-23], which provide important information regarding surface and nanostructure evolution, cannot provide detailed atomistic dynamics and kinetics, due to the timescales involved, that govern the initial stages of nucleation leading to film growth.

Ab initio calculations focusing on atomic diffusion on TM nitride surfaces are commonly used to determine minimum energy paths and corresponding diffusion barriers at $0 \mathrm{~K}$ [24-26]. Generally, this is accomplished by determining adspecies static adsorption-energy landscapes, or using algorithms such as the nudged elastic band (NEB) method [27,28]. However, the rate of thermally-activated migration events depends not only on diffusion barriers, but also on jump 
attempt frequencies [29]. Vineyard showed that the latter can be estimated within the harmonic approximation at $0 \mathrm{~K}$ from the ratio of the product of the $\mathrm{N}$ phonon frequencies at the starting point of the transition to the $(\mathrm{N}-1)$ phonon frequencies of the system constrained in the transition-state saddle-point configuration $[30,31]$.

While providing estimates of diffusion barriers and attempt frequencies, the computational approaches described above do not account for the fact that activation energies and pathways are affected by atomic vibrations at finite temperatures. Moreover, Arrhenius exponential prefactors estimated from $0 \mathrm{~K}$ phonon calculations do not accurately describe diffusion attempt-frequencies for materials characterized by large anharmonic contributions to atomic interactions. The approximation proposed by Vineyard [31] is not applicable to TM nitride compounds, such as B1 $\mathrm{NaCl}$-structure vanadium nitride, which are predicted to be dynamically unstable at $0 \mathrm{~K}$ [32]. Molecular dynamics (MD) simulations are the most accurate and reliable approaches available to determine surface diffusion jump rates and pathways at finite temperatures.

In MD simulations, the atomic interactions can be described by first-principles or empirical formulations; both methods have advantages and disadvantages. Density functional theory (DFT) is, in principle, the most accurate methodology available to calculate interatomic forces during MD runs [33]. However, DFT has several limitations [34]. The approximations used in DFT for estimation of electron exchange and correlation energy are optimized to describe three-dimensional lattices. However, the abrupt changes which occur in the electron density at surfaces can result in under- or overestimation of surface/adsorbate bond strengths, and hence incorrect adsorption energies [35]. This, in turn, results in incorrect predictions for the relative stabilities of different adsorption sites [36] and, in DFT-based ab initio molecular dynamics (AIMD) simulations of surface kinetics, in incorrect predictions of preferred adsorbate diffusion pathways and jump rates.

Highly computationally demanding AIMD/DFT runs are also limited to the simulation of small systems (a few hundred atoms) and for short time intervals (a few nanoseconds). This does 
not allow investigation of the effects of phonon wavelength degrees of freedom on atomic migration, estimation of the effect of correlated jumps on diffusion coefficients, or obtaining wellconverged values of rate constants for rare events. These limitations are easily overcome when, instead of DFT, empirical potentials are used for the description of interatomic forces. However, classical molecular dynamics (CMD) simulations based upon empirical potentials are less accurate than quantum-mechanical approaches. Despite their individual limitations, AIMD and CMD simulations can be used in parallel to provide complementary information. When AIMD and CMD results are consistent, it is reasonable to assume that the agreement is not accidental, and that the theoretical predictions are realistic representations of actual physical phenomena.

In this study, we probe the dynamics of $\mathrm{Ti}$ adatoms on $\mathrm{TiN}(001)$ as a function of temperature by performing both AIMD simulations based on DFT and CMD simulations in which the interatomic forces are obtained from the modified embedded atom method (MEAM) potential parameterized to reproduce TiN bulk, as well as surface, properties [37-39]. Titanium nitride, one of the first hard-coating materials $[15,40,41]$, serves as a model to probe diffusion dynamics and kinetics on NaCl-structure TM nitride surfaces in general.

AIMD/DFT and CMD/MEAM simulations show that $\mathrm{Ti}$ adatoms on $\mathrm{TiN}(001)$ favor fourfold-hollow positions, surrounded by two Ti and two $\mathrm{N}$ surface atoms; adatom migration between these sites occurs primarily via in-plane $<100>$ channels. We determine activation energies $\mathrm{E}_{\mathrm{a}}$ and jump-attempt frequencies $\mathrm{A}$ of the most frequently observed Ti adatom diffusion pathways on $\operatorname{TiN}(001)$ extrapolated from jump-rate $\ln (\mathrm{k})$ vs. inverse temperature $1 / \mathrm{T}$ Arrhenius plots, in which $\mathrm{k}$ is the rate constant. We assess the effect of increasing phonon wavelength degrees of freedom by using progressively larger simulation supercells in CMD runs. Finally, the Ti adatom normal distributions on TiN(001), obtained using uncorrelated jump probabilities Aexp[- $\left.\mathrm{E}_{\mathrm{a}} /\left(\mathrm{k}_{\mathrm{B}} \mathrm{T}\right)\right]$, are employed to determine the surface diffusion coefficient $\mathrm{D}_{\mathrm{s}}$ as a function of temperature $\mathrm{T}$. The latter values are compared to those extracted directly from the adatom mean-square displacements 
during CMD runs to evaluate correlation between consecutive jumps. Remarkably, diffusion coefficient results obtained from AIMD and CMD are in good agreement at all temperatures.

\section{Computational details}

MD simulations are performed within the microcanonical ensemble NVE, while the substrate temperature is maintained constant via periodic rescaling of the atomic velocities, which mimics a canonical NVT sampling of configurational space [42]. Standard Verlet algorithms are used to integrate Newton's equations of motion at $1 \mathrm{fs}$ time intervals for both CMD and AIMD runs. CMD simulations are carried out by describing atomic interactions with the second-neighbor MEAM [43] potential as implemented in the large-scale atomic/molecular massively parallel simulator (LAMMPS) [44]. We use the TiN MEAM parameters from our previous publications [37-39,45], which were shown to yield diffusion barriers, Ehrlich step-edge barriers, surface formation energies, step-edge formation energies, and adatom formation energies which are consistent with experimental values $[18,20,46]$, as well as a description of single $\mathrm{N}$ adatom and $\mathrm{N}$ adatom pair interactions on $\operatorname{TiN}(001)$ which are in agreement with AIMD/DFT simulation predictions [39]. Ab initio molecular dynamics simulations are carried out with the VASP code [47] implemented using the generalized gradient approximation (GGA) [48] and the projector augmented wave (PAW) [49] method. The Brillouin zone is sampled with $3 \times 3 \times 1$ k-point grids centered at the $\Gamma$ point. At each time step, the total energy is evaluated to an accuracy of $10^{-5}$ eV/atom using a plane-wave energy cutoff of $400 \mathrm{eV}$.

Ti adatom diffusion pathways and jump rates are probed as a function of temperature on $3 \times 3$ TiN(001) surface unit cells. The substrate consists of three layers for a total of 108 atoms in AIMD runs, and eight layers with 288 atoms in CMD runs. The lateral size of the simulation cell is sufficient to avoid adatom self-interactions. During all MD runs, the bottom slab layer remains fixed. This does not affect the substrate temperature, which depends only on the atomic-motion translational degrees of freedom. AIMD simulation boxes contain six vacuum layers to prevent 
interaction between $\mathrm{TiN}(001)$ surface slab replicas along the [001] direction. At each temperature, the average $\mathrm{Ti}-\mathrm{N}$ in-plane nearest-neighbor distance $<\mathrm{d}_{\mathrm{NN} / /}>$ in the simulation slab is obtained, accounting for the experimental TiN thermal expansion coefficient $9.35 \times 10^{-6} \mathrm{~K}^{-1}$ [4], by rescaling the $0 \mathrm{~K}<\mathrm{d}_{\mathrm{NN} / /}>$ values, $2.127 \AA$ for DFT+GGA [50] and 2.121 $\AA$ for MEAM [37]. Rescaling of $<\mathrm{d}_{\mathrm{NN} / /}>$ as a function of temperature is necessary to avoid spurious substrate strain effects on the adatom jump rate [51]. Prior to initiating each MD run, thermal oscillations in the adatom-free simulation slab are allowed to stabilize for five ps, a time interval sufficient to equilibrate the phonon modes in the system.

Ten statistically independent CMD runs of $10 \mathrm{~ns}$ are performed at temperatures $\mathrm{T}$ of 600 , 900, 1200 , and $1500 \mathrm{~K}$ for a total of $0.4 \mu$ s simulated time. 0.5-ns AIMD runs are performed at $1000,1200,1500$, and $2200 \mathrm{~K}$, for a total of 2 ns simulation time. All results are stored in video files with a time resolution of $10 \mathrm{fs}$. In order to further verify the reliability of our AIMD model, we vary the number of simulation slab layers from three to five (180 atoms) to test the dependence of the diffusion rate constant $k$ on substrate thickness. Two 40-ps AIMD runs, performed at $\mathrm{T}=2200$ and $2500 \mathrm{~K}$ with the larger simulation cell, return jump-rates which are in good agreement with those calculated for three-layer slabs.

We use MEAM and DFT conjugate gradient minimizations to calculate Ti adatom adsorption energy landscapes on $4 \times 4$ atom static TiN(001) supercells consisting of five layers, by sampling the irreducible triangle of the surface unit cell on a 25-point grid. This is accomplished by relaxing the upper two $\mathrm{TiN}(001)$ layers and the vertical coordinates of the adspecies at each point. Adsorption energies are evaluated to an accuracy of $10^{-5} \mathrm{eV} /$ atom, which in DFT is achieved by using a plane-wave cutoff of $500 \mathrm{eV}$ and integrating the Brillouin zone on 15x15x1 k-point meshes centered at $\Gamma$. Both quantum-mechanical and empirical results indicate that the most stable position for isolated $\mathrm{Ti}$ adatoms on infinite $\mathrm{TiN}(001)$ terraces at $0 \mathrm{~K}$ is in fourfold hollow sites surrounded by two Ti and two $\mathrm{N}$ surface atoms (Fig. 1). Considering the uncertainty regarding the accuracy of 
DFT for surface calculations $[35,36,52]$, the agreement between DFT + GGA and MEAM predictions (see adsorption energy values in Fig. 1) for Ti monomer stability at various surface sites is quite good.

In order to determine the preferred adsorption position of $\mathrm{Ti}$ adatoms on $\operatorname{TiN}(001)$ at temperatures between 1000 and $2200 \mathrm{~K}$, we perform preliminary AIMD tests in which the initial adatom (x,y) coordinates are randomly chosen at a distance of $\sim 2 \AA$ above the surface. In all cases, Ti adatoms quickly reach and remain in fourfold hollow sites, in accordance with the predictions of our present $0 \mathrm{~K} \mathrm{DFT}$ and MEAM calculations, and previous CMD simulations at $1000 \mathrm{~K}$ [37]. Thus, a fourfold hollow site is chosen as the starting position for Ti adatoms in all MD runs.

For each Ti adatom diffusion pathway $\mathrm{m}\left[\overrightarrow{\mathbf{r}}_{\mathbf{i}} \rightarrow \overrightarrow{\mathbf{r}}_{\mathbf{i}}+\overrightarrow{\mathbf{r}}_{\mathbf{j}_{-}}\right.$(for simplicity, expressed as $m(i \rightarrow j))$ between neighboring $\mathrm{i}$ and $\mathrm{j}$ fourfold hollow sites on $\mathrm{TiN}(001)$ terraces, we determine activation energies $E_{a}$ and attempt frequencies $A$ by linear interpolation of the logarithm of the jump rate $\mathrm{k}(\mathrm{T})$ (obtained from $\mathrm{MD}$ runs as a function of $\mathrm{T}$ ) vs. inverse temperature as prescribed by the Arrhenius equation:

$$
\ln \left[k^{m(i \rightarrow j)}(T)\right]=\ln \left[A^{m(i \rightarrow j)}\right]-\frac{E_{a}^{m(i \rightarrow j)}}{k_{B}}\left(\frac{1}{T}\right) .
$$

The uncertainties $\sigma_{\kappa}, \sigma_{\mathrm{A}}$, and $\sigma_{\mathrm{E}}$, associated with the values of rate coefficient $\ln (\mathrm{k})$, attempt frequency $\mathrm{A}$, and activation energy $\mathrm{E}_{\mathrm{a}}$, are estimated following the scheme reported in reference [39].

Ti adatom surface diffusion coefficients $\mathrm{D}_{\mathrm{s}}$ on $\mathrm{TiN}(001)$ are determined from the normalized probability distribution function $\mathbf{P}(\vec{r}, t, T)$, which corresponds to the probability of finding the adspecies in a (001) surface unit cell centered at position $\overrightarrow{\mathbf{r}}$ at time $\mathrm{t}$ and temperature $\mathrm{T}$, using the two-dimensional Einstein relation: 


$$
\mathrm{D}_{\mathrm{s}}(\mathrm{T})=\lim _{\mathrm{t} \rightarrow \infty} \frac{\left\langle\left[\sum_{\overrightarrow{\mathrm{r}}} \mathrm{P}(\overrightarrow{\mathrm{r}}, \mathrm{t}, \mathrm{T}) \cdot|\overrightarrow{\mathrm{r}}|^{2}\right\rangle\right.}{4 \mathrm{t}} .
$$

The summation in equation (2) extends over all (001) surface lattice vectors $\overrightarrow{\mathbf{r}}$, and $\mathrm{P}$ at time $\mathrm{t}=0$ is a Dirac delta function centered at the adatom initial position $\overrightarrow{\mathbf{r}}=0$. Eq. (2) yields diffusion coefficients corresponding to adatom random walks (uncorrelated jumps) on $\mathrm{TiN}(001)$.

$\mathrm{P}(\overrightarrow{\mathbf{r}}, \mathbf{t}, \mathbf{T})$ can be calculated using transition probabilities from sites $\mathrm{i}$ to sites $\mathrm{j}$ : $\mathrm{A}^{\mathrm{m}(\mathrm{i} \rightarrow \mathrm{j})} \exp \left[-\mathrm{E}_{\mathbf{a}}^{\mathrm{m}(\mathrm{i} \rightarrow \mathrm{j})} /\left(\mathrm{k}_{\mathrm{B}} \mathrm{T}\right)\right]$. At any temperature $\mathrm{T}, \mathrm{P}$ at time $(\mathrm{t}+\Delta \mathrm{t})$ is obtained from $\mathrm{P}$ at time $\mathrm{t}$ plus an inward flux $\Phi_{\text {in }}$, i.e. the probability for the adatom $m(i \rightarrow j)$ migration from neighboring surface unit cells into the fourfold hollow site at position $\overrightarrow{\mathbf{r}}$, minus an outward transition probability $\Phi_{\text {out }}$ due to adspecies moving from position $\overrightarrow{\mathbf{r}}$ to neighboring sites via $\mathrm{m}(\mathrm{i} \rightarrow \mathbf{j})$. Thus,

$$
\mathrm{P}(\overrightarrow{\mathrm{r}}, \mathrm{t}+\Delta \mathrm{t}, \mathrm{T})=\mathrm{P}(\overrightarrow{\mathrm{r}}, \mathrm{t}, \mathrm{T})+\Phi_{\text {in }}-\Phi_{\text {out }},
$$

for which

$$
\Phi_{\text {in }}=\sum_{\vec{r}_{j}}\left\{P\left(\vec{r}+\vec{r}_{j}, t, T\right) A^{m(i \rightarrow j)} \exp \left[-E_{a}^{m(i \rightarrow j)} /\left(k_{B} T\right)\right] \Delta t\right\}
$$

and

$$
\Phi_{\text {out }}=\sum_{\vec{r}_{j}}\left\{\mathrm{P}(\overrightarrow{\mathrm{r}}, \mathrm{t}, \mathrm{T}) \mathrm{A}^{\mathrm{m}(\mathrm{i} \rightarrow \mathrm{j})} \exp \left[-\mathrm{E}_{\mathrm{a}}^{\mathrm{m}(\mathrm{i} \rightarrow \mathrm{j})} /\left(\mathrm{k}_{\mathrm{B}} \mathrm{T}\right)\right] \Delta \mathrm{t}\right\}
$$

The summations in Eqs. (4) and (5) extend over all $\vec{r}_{j}$ vectors connecting neighboring surface fourfold hollow sites to which the adatom can migrate via the diffusion mechanism $m(i \rightarrow j)$. The normalization of $\mathrm{P}$ is preserved at any given time $\mathrm{t}$ provided that the interval $\Delta \mathrm{t}$ is much smaller than the inverse jump rate $1 / \mathrm{k}$ at temperature $\mathrm{T}$. The uncertainty $\left|\sigma_{\mathrm{D}}(\mathrm{T})\right|$ associated with surface 
diffusion coefficients values $\mathrm{D}_{\mathrm{s}}(\mathrm{T})$ deriving from stochastic Ti adatom walks on $\mathrm{TiN}(001)$ is obtained from the relationship: $\left|\sigma_{\mathrm{D}}(\mathrm{T})\right|=\left|\mathrm{D}_{\max }(\mathrm{T})-\mathrm{D}_{\min }(\mathrm{T})\right| / 2$. Upper and lower boundary values, $\mathrm{D}_{\max }(\mathrm{T})$ and $\mathrm{D}_{\min }(\mathrm{T})$, of the diffusion coefficient are obtained from Eqs. (2) - (5) by applying the substitutions $\left[\mathrm{A} \rightarrow \mathrm{A}+\sigma_{\mathrm{A}} / \sqrt{ } 2 ; \mathrm{E}_{\mathrm{a}} \rightarrow \mathrm{E}_{\mathrm{a}}-\sigma_{\mathrm{E}} / \sqrt{ } 2\right]$ and $\left[\mathrm{A} \rightarrow \mathrm{A}-\sigma_{\mathrm{A}} / \sqrt{ } 2 ; \mathrm{E}_{\mathrm{a}} \rightarrow \mathrm{E}_{\mathrm{a}}+\sigma_{\mathrm{E}} / \sqrt{ } 2\right]$, respectively.

After a sufficiently long time $\mathrm{t} \gg \mathrm{1} / \mathrm{k}, \mathbf{P}(\overrightarrow{\mathbf{r}}, \mathbf{t}, \mathbf{T})$ becomes a uniform radial distribution function $\overline{\mathbf{P}}(\mathrm{r}, \mathrm{t}, \mathrm{T})$. Thus, at time $\mathrm{t}$ and temperature $\mathrm{T}$, the normalized probability $\overline{\mathbf{P}}(\mathrm{r}, \mathrm{t}, \mathrm{T})$ to find a Ti adatom at a distance $r$ from its initial position is

$$
\bar{P}(r, t, T)=\frac{P(\vec{r}, t, T) \cdot 2 \pi r \Delta r}{\sum_{r}[P(\vec{r}, t, T) \cdot 2 \pi r \Delta r]},
$$

and the average adatom displacement $\langle\boldsymbol{\alpha}(\mathrm{t}, \mathrm{T})\rangle$ can be obtained from:

$$
\langle\alpha(\mathrm{t}, \mathrm{T})\rangle=\sum_{\mathrm{r}}[\mathrm{r} \cdot \overline{\mathrm{P}}(\mathrm{r}, \mathrm{t}, \mathrm{T}) \Delta \mathrm{r}]=\sqrt{4 \mathrm{t} \mathrm{D}_{\mathrm{s}}(\mathrm{T})}
$$

By linear interpolation of $\ln \left[\mathrm{D}_{\mathrm{s}}(\mathrm{T})\right]$ vs. $1 / \mathrm{T}$, we extract convolved values (since Ti adatom diffusion occurs via more than one pathway) of Arrhenius prefactors $\mathbf{A}^{\prime}$ and diffusion barriers $\mathbf{E}_{\mathrm{a}}^{\prime}$, which correspond to the radial (isotropic) diffusion of Ti adspecies on $\operatorname{TiN}(001)$ surfaces:

$$
\ln \left[\mathrm{D}_{\mathrm{s}}(\mathrm{T})\right]=\ln \left(\mathrm{A}^{\prime}\right)-\frac{\mathrm{E}_{\mathrm{a}}^{\prime}}{\mathrm{k}_{\mathrm{B}}}\left(\frac{1}{\mathrm{~T}}\right)
$$

d of $\sigma_{D}(T)$ affects those of the uncertainties $\sigma_{A}^{\prime}$ and $\sigma_{E}^{\prime}$, which are associated with the values of $\mathbf{A}^{\prime}$ and $\mathrm{E}_{\mathrm{a}}^{\prime}$, respectively. $\sigma_{\mathrm{A}}^{\prime}$ and $\sigma_{\mathbf{E}}^{\prime}$ are assessed by following the scheme described previously [39]. 
In CMD runs, we progressively enlarge the simulation slab, from $3 \times 3$ to $5 \times 5,8 \times 8,12 \times 12$, and $17 \times 17$ surface unit cells (for a total of 288, 800, 2048, 4608, and 9248 atoms, respectively), to investigate the effect on $\mathrm{Ti}$ adatom mobility of increasing the phonon wavelength degrees of freedom. We perform ten 10-ns CMD runs, at each of the temperatures listed above for each supercell size, yielding a total simulated time of $2 \mu \mathrm{s}$, and find that a $17 \times 17$ unit cell surface area is sufficient to achieve saturation of calculated $\mathrm{A}^{\prime}$ and $\mathrm{E}_{\mathrm{a}}^{\prime}$ values.

Finally, $\mathrm{D}_{\mathrm{s}}(\mathrm{T})$ results obtained by multiplying uncorrelated transition probabilities (Equations $(2)-(5))$ are compared with $\mathrm{D}_{\mathrm{s}}^{\mathrm{cor}}(\mathrm{T})$ values calculated from the two-dimensional Einstein relation:

$$
D_{s}^{\text {corr }}(\mathrm{T})=\lim _{\mathrm{t} \rightarrow \infty} \frac{\left\langle[\overrightarrow{\mathrm{r}}(\mathrm{t})-\overrightarrow{\mathrm{r}}(0)]^{2}\right\rangle}{4 \mathrm{t}},
$$

for which adspecies mean-square displacements are tracked during 10-ns CMD simulations on large $\operatorname{TiN}(001)$ terraces $(8 \times 8,12 \times 12$, and $17 \times 17$ surface unit cells $)$, at the temperatures listed above, for a total of 120 runs. We also use data collected during our previous CMD investigations [37] of Ti adatom dynamics on 9x9 surface unit cell TiN(001) simulation slabs at $1000 \mathrm{~K}$. The comparison between $D_{s}(T)$ and $D_{s}^{\text {corr }}(T)$ values allows us to estimate the effect of correlated jumps on the diffusion coefficient. At any given temperature, the uncertainty $\sigma_{\mathbf{D}}^{\text {corr }}(\mathrm{T})$ associated with $\mathrm{D}_{\mathbf{s}}^{\mathrm{cor}}$ values is calculated via the relationship: $\sigma_{\mathrm{D}}^{\mathrm{cor}}(\mathrm{T})=\left[\ln \left(\overline{\mathrm{D}}_{\mathrm{s}}^{\mathrm{cor}}(\mathrm{T}) / \mathrm{D}_{\min }^{\mathrm{cor}}(\mathrm{T})\right)+\ln \left(\mathrm{D}_{\max }^{\mathrm{cor}}(\mathrm{T}) / \overline{\mathrm{D}}_{\mathrm{s}}^{\mathrm{cor}}(\mathrm{T})\right)\right] / 2[53]$, for which the average, $\overline{\mathrm{D}}_{\mathrm{s}}^{\mathrm{cor}}(\mathrm{T})$ and the extreme, $\mathrm{D}_{\min }^{\mathrm{cor}}(\mathrm{T})$ and $\mathrm{D}_{\max }^{\mathrm{cor}}(\mathrm{T})$, values of the diffusion coefficient are determined from 30 CMD runs. $\mathrm{D}_{\mathrm{s}}^{\mathrm{corr}}$ values outside the range $\left[\mathrm{D}_{\min }^{\mathrm{cor}}(\mathrm{T}), \mathrm{D}_{\max }^{\mathrm{cor}}(\mathrm{T})\right]$ are unlikely. Activation energies $\mathrm{E}_{\mathrm{a}}^{\prime \text { corr }} \pm \sigma_{\mathbf{E}}^{\prime}$ and exponential prefactors $\mathrm{A}^{\prime \text { corr }} \pm \sigma_{\mathrm{A}}^{\prime}$, corresponding to correlated/isotropic Ti adatom 
surface diffusion, are obtained in analogy with uncorrelated isotropic values.

\section{Results and discussion}

MD movies show that in-plane $<100>$ and $<110>$ translations (Fig. 2), the latter passing atop a $\mathrm{N}$ surface atom, are the preferred diffusion pathways for Ti adatoms migrating between neighboring fourfold-hollow sites on $\operatorname{TiN}(001)$.

In $\mathrm{CMD}$ runs, $\mathrm{Ti}<100>$ migration is slightly bowed; the adatom passes closer to $\mathrm{N}$ than to Ti while crossing the boundary between nearest-neighbor surface unit cells. This is consistent with the curvature of the adsorption energy landscapes obtained from MEAM $0 \mathrm{~K}$ calculations (Fig. 1), which show that the Ti adatom minimum energy path connecting neighboring fourfold-hollow sites crosses the Ti-N surface-atom bond via a saddle point located nearer to $\mathrm{N}$. The latter is due to the higher affinity of the $\mathrm{Ti}$ adatom for $\mathrm{N}$ than for Ti surface atoms. Ti adatom $<110>$ jumps always occur with the adspecies passing atop metastable epitaxial sites, where they spend up to a few femtoseconds.

In AIMD simulations, Ti adatoms always transit through metastable epitaxial positions during migration on $\operatorname{TiN}(001)$. Once atop a $\mathrm{N}$ surface atom, where the adatom resides for a few fs, it can return to the initial stable site (resulting in no net migration), proceed along the same $<110>$ direction, or move orthogonal to the initial motion, thus yielding a net $<100>$ translation. This result is consistent with DFT $0 \mathrm{~K}$ adsorption energy landscapes (see Fig. 1 and references [24,25]), showing that the minimum energy path connecting neighboring fourfold hollow sites is across $\mathrm{N}$ atop positions.

Both CMD and AIMD runs reveal that Ti adatoms can also perform $<100>$ and $<110>$ double jumps on $\mathrm{TiN}(001)$ terraces. $<100>$ double jumps are the second most frequently recorded diffusion mechanism during CMD runs. For this reason, double-jump attempt frequencies and activation energies are calculated separately. In AIMD runs, double jumps take place along $<110>$ 
more often than along $<100>$. However, due to limited AIMD simulation times, it is not possible to determine accurate rate coefficients for long jumps, which are instead counted as multiple singlemigration events.

Fig. 3 shows CMD and AIMD Arrhenius plots of the temperature dependent total jump rate $\mathrm{k}_{\text {tot }}$ of $\mathrm{Ti}$ adatoms migrating on $\mathrm{TiN}(001)$. To obtain $\mathrm{k}_{\text {tot }}$, each double jump corresponds to two migration events. $k_{\text {tot }}$ obtained from AIMD runs also includes diffusion events in which an adatom moves from a fourfold hollow site to an epitaxial site, and then returns to the initial position. Remarkably, CMD and AIMD simulations provide very similar diffusion rates. From the total number of migration events, we extract $E_{a}=(0.51 \pm 0.03) \mathrm{eV}$ and $\mathrm{A}=(0.79 \pm 0.14) \times 10^{13} \mathrm{~s}^{-1}$ for AIMD, and $\mathrm{E}_{\mathrm{a}}=(0.69 \pm 0.02) \mathrm{eV}$ and $\mathrm{A}=(5.30 \pm 0.80) \times 10^{13} \mathrm{~s}^{-1}$ for CMD. We note that the AIMD activation energy of $0.51 \mathrm{eV}$ is almost $50 \%$ higher than that obtained from DFT/NEB calculations on static $\operatorname{TiN}(001)$ terraces of the same size [24]. A similar difference was observed when comparing the barriers estimated from DFT/NEB and AIMD/DFT for $\mathrm{N}$ adatom diffusion on $\operatorname{TiN}(001)$ [39]. These results highlight the fact that lattice vibrations strongly affect the energetics of adatom diffusion on $\operatorname{TiN}(001)$.

Fig. $4 \mathrm{a}$ is a plot of $\mathrm{Ti}$ adatom jump rates as a function of $\mathrm{T}$ for the two migration pathways observed during AIMD runs, i.e. $<100>$ and $<110>$ single jumps. The AIMD simulations result in a linear Arrhenius $\ln (\mathrm{k})$ vs. 1/T behavior for both diffusion mechanisms. From the interpolation of $\ln (\mathrm{k})$ data points, using Eq. (1), we extract $\mathrm{E}_{\mathrm{a}}=(0.54 \pm 0.06) \mathrm{eV}$ and $\mathrm{A}=(0.53 \pm 0.22) \times 10^{13} \mathrm{~s}^{-1}$ for $<100>$ single jumps and $E_{a}=(0.44 \pm 0.07) \mathrm{eV}$ and $\mathrm{A}=(0.15 \pm 0.08) \times 10^{13} \mathrm{~s}^{-1}$ for $<110>$ jumps. The trends for $\ln (\mathrm{k})$ values vs. $1 / \mathrm{T}$ suggest that $<100>$ jumps occur more frequently than $<110>$ jumps for $\mathrm{T} \gtrsim 1000 \mathrm{~K}$, while $<110>$ migration becomes the most likely mechanism at $\mathrm{T} \lesssim 1000 \mathrm{~K}$. Nevertheless, since AIMD uncertainties $\sigma_{\kappa}(\mathrm{T})$ are large with respect to the difference between $<100>$ and $<110>$ diffusion rates (see error bars in Fig. 4a), it is not possible to provide accurate 
estimates of the relative occurrence probability of $<100>$ and $<110>$ hops as a function of temperature.

CMD simulations predict that $<100>$ migration is the preferred $\mathrm{Ti}$ adatom pathway on $\operatorname{TiN}(001)$ at all temperatures. $<100>$ single jumps are the most frequently observed diffusion events, followed by $<100>$ double jumps, and $<110>$ single jumps (see Fig. 4 b); activation energies and attempt frequencies are $\mathrm{E}_{\mathrm{a}}=(0.66 \pm 0.03),(0.88 \pm 0.04),(0.95 \pm 0.06) \mathrm{eV}$ and $\mathrm{A}=(2.80 \pm$ $0.80),(3.07 \pm 1.05),(1.61 \pm 0.73) \times 10^{13} \mathrm{~s}^{-1}$, respectively. The ratio between the rates of $<100>$ double and single jumps increases with T. Double jumps account for approximately $5 \%$ of the total number of $<100>$ migration events at $900 \mathrm{~K}$ and $25 \%$ at $1500 \mathrm{~K}$. During CMD simulations performed at 1200 and $1500 \mathrm{~K}$, we also observe Ti adatom $<100>$ triple jumps and $<110>$ double jumps. These, however, account for only $0.1 \%$ of the total number of diffusion events. The fact that long jumps become statistically more relevant with increasing $\mathrm{T}$ is consistent with AIMD results, in which double jumps occur only at $\mathrm{T} \geq 1500 \mathrm{~K}$, with the highest probability, approximately $20 \%$ of the total number of diffusion events, obtained during simulations performed at $2200 \mathrm{~K}$.

In CMD runs performed at $1500 \mathrm{~K}$, we also observe $\mathrm{Ti}$ adatom diffusion occurring by exchange with a Ti surface atom. The Ti adatom moves downward into the TiN(001) substrate with a frequency of approximately 30 events per $\mu$ s. The strain induced in the lattice by the migration of an interstitial Ti atom propagates horizontally within the (001) plane until one of the Ti atoms in the outermost slab layer moves upward onto the surface, thus becoming an adatom. However, this pathway is not observed in our AIMD simulations due to the very low occurrence frequency.

AIMD simulations are confined, because of the high computational cost, to the study of relatively small supercells. This limits the possibility of probing the effect of long wavelength phonons on adatom mobility. However, such effects can be studied in CMD runs. Fig. 5 shows Arrhenius plots of total jump rates vs. 1/T recorded during CMD simulations of Ti adatoms migrating on $\mathrm{TiN}(001)$ eight-layer supercells with progressively increasing lateral sizes: $3 \times 3$, 5x5, 
$8 \times 8,12 \times 12$, and $17 \times 17$ surface unit cells. While $\mathrm{k}$ increases significantly (by a factor of 2.5 ) with supercell area at $600 \mathrm{~K}$, its percentage variation is negligible at higher temperatures. Thus, longwavelength lattice vibrations promote significantly adsorbate mobility only at lower temperatures.

The inserts in Fig. 5 are plots of CMD-calculated activation energies $E_{a}$ and attempt frequencies A, corresponding to the total number of diffusion events, as a function of the supercell surface area. $E_{a}$ and $A$ reach asymptotic values of $(0.62 \pm 0.01) \mathrm{eV}$ and $(2.76 \pm 0.23) \times 10^{13} \mathrm{~s}^{-1}$ for the largest of the supercells $\left(17 \times 17\right.$ surface unit cells). We note that the same $E_{a}$ and $A$ values would be obtained for small supercells ( $3 \times 3$ surface unit cells) by linear interpolation of the CMD Ti adatom total jump rates at temperatures $\geq 900 \mathrm{~K}$. Thus, at high $\mathrm{T}$, the number of diffusion events per unit time converges rapidly with supercell size. This suggests that the AIMD jump rate values shown in Figs. 3 and $4 a$, with a corresponding activation energy $E_{a}=(0.51 \pm 0.03) \mathrm{eV}$ and attempt frequency $\mathrm{A}=(0.79 \pm 0.14) \times 10^{13} \mathrm{~s}^{-1}$, are saturated with respect to supercell surface area.

The higher phonon-mode degrees of freedom, achieved by increasing the surface area from $3 \times 3$ to $17 \times 17$ unit cells, affects the occurrence probability of all diffusion pathways. Ti adatom $<100>$ single jumps are still the most frequently recorded events. $<100>$ jump activation energies and attempt frequencies calculated for small supercells, $E_{a}^{3 \times 3}=(0.66 \pm 0.03) \mathrm{eV}$ and $\mathrm{A}^{3 \times 3}=(2.80 \pm$ $0.80) \times 10^{13} \mathrm{~s}^{-1}$, both decrease for large simulation slabs; $E_{a}^{17 \times 17}=(0.57 \pm 0.01) \mathrm{eV}$ and $\mathrm{A}^{17 \times 17}=$ $(1.09 \pm 0.10) \times 10^{13} \mathrm{~s}^{-1}$. At $600 \mathrm{~K},<100>$ single-jump rates increase by a factor of 2.6 , while a slight decrease of $12 \%$ is observed at $1500 \mathrm{~K}$ because of the increasingly significant fraction of long jumps. Due to lattice vibrations with larger wavelength, the $<100>$ double-jump activation energy decreases from $E_{a}^{3 \times 3}=(0.88 \pm 0.04) \mathrm{eV}$ to $E_{a}^{17 \times 17}=(0.84 \pm 0.01) \mathrm{eV}$, while the corresponding attempt frequency increases from $\mathrm{A}^{3 \times 3}=(3.07 \pm 1.05) \times 10^{13} \mathrm{~s}^{-1}$ to $\mathrm{A}^{17 \times 17}=(3.36 \pm 0.15) \times 10^{13} \mathrm{~s}^{-1}$. Overall, the occurrence of double jumps increases at all temperatures. Ti adatom $<100>$ double jumps are never observed during $0.1 \mu \mathrm{s}$ CMD movies at $600 \mathrm{~K}$. However, the jump rate estimated 
at $900 \mathrm{~K}$ for small supercells, $\mathrm{k}^{3 \times 3}=(3.7 \pm 0.2) \times 10^{8} \mathrm{~s}^{-1}$, increases by $80 \%$ for large simulation substrates, $\mathrm{k}^{17 \times 17}=(6.6 \pm 0.1) \times 10^{8} \mathrm{~s}^{-1}$, and by $45 \%$ at $1500 \mathrm{~K}: \mathrm{k}^{3 \times 3}=(3.5 \pm \varepsilon) \times 10^{10} \mathrm{~s}^{-1}$ and $\mathrm{k}^{17 \times 17}$ $=(5.1 \pm \varepsilon) \times 10^{10} \mathrm{~s}^{-1}$, in which $\varepsilon<<0.1$.

As was the case for $3 \times 3$ surface unit cells, $<110>$ single jumps are the least frequently observed diffusion mechanisms for $17 \times 17$ unit cells. Nevertheless, $<110>$ jump rates increase significantly on large supercells; the corresponding activation energy and attempt frequency range from $E_{a}^{3 \times 3}=(0.95 \pm 0.06) \mathrm{eV}$ and $\mathrm{A}^{3 \times 3}=(1.61 \pm 0.73) \times 10^{13} \mathrm{~s}^{-1}$ to $E_{a}^{17 \times 17}=(0.81 \pm 0.03) \mathrm{eV}$ and $\mathrm{A}^{17 \times 17}=(0.51 \pm 0.14) \times 10^{13} \mathrm{~s}^{-1}$. AIMD and CMD estimates of diffusion barriers $\mathrm{E}_{\mathrm{a}}$ and jump attempt frequencies A for the three primary Ti adatom pathways on $\mathrm{TiN}(001)$ are summarized in Table 1. We note that uncertainties $\sigma_{\mathrm{A}}$ and $\sigma_{\mathrm{E}}$ are considerably reduced upon increasing the supercell size, as diffusion rates $\ln \left(\mathrm{k}^{17 \times 17}\right)$ vary with $1 / \mathrm{T}$ in a more linear fashion than for $3 \times 3$ supercells. At $900 \mathrm{~K}, \mathrm{k}^{17 \times 17}$ is $2 \mathrm{x}$ larger than $\mathrm{k}^{3 \times 3}$; the difference decreases to $\sim 5 \%$ larger at $1500 \mathrm{~K}$. The vibrational degrees of freedom for $34 \times 34$ atom $\operatorname{TiN}(001)$ simulation layers yield saturated values of Ti adatom total jump rates, which are higher than those recorded on small supercells by $160 \%$ at $600 \mathrm{~K}\left(\boldsymbol{k}_{\text {tot }}^{3 \times 3}=(0.7 \pm 0.2) \times 10^{8} \mathrm{~s}^{-1}\right.$ and $\left.\boldsymbol{k}_{\text {tot }}^{17 \times 17}=(1.8 \pm 0.2) \times 10^{8} \mathrm{~s}^{-1}\right)$, and by $7 \%$ at $1500 \mathrm{~K}$ $\left(\boldsymbol{k}_{\text {tot }}^{3 \times 3}=(21.8 \pm \varepsilon) \times 10^{10} \mathrm{~s}^{-1}\right.$ and $\boldsymbol{k}_{\text {tot }}^{17 \times 17}=(23.4 \pm \varepsilon) \times 10^{10} \mathrm{~s}^{-1}$, where $\left.\varepsilon<<0.1\right)$.

Diffusion coefficients, which provide a well-defined measure of atomic mobility, depend upon both jump activation energies and attempt frequencies. Nevertheless, theoretical studies of atomic diffusion generally focus only on determination of diffusion barriers [24-26,54]. Using Eqs. (2) - (5), parameterized with the saturated values of activation energies and attempt frequencies obtained from AIMD and CMD jump rates for the different diffusion pathways, Ti adatom diffusion coefficients $\mathrm{D}_{\mathrm{s}}$ on $\mathrm{TiN}(001)$ are evaluated as a function of temperature (see Fig. 6). Regardless of the fact that $a b$ initio and classical methods predict different Ti adatom $<100>$ and $<110>$ jump 
rates, AIMD and CMD diffusion coefficients based purely on uncorrelated hops (random walk) are in good agreement over the investigated temperature range (see Fig. 6 and Table 2).

An accurate evaluation of the $\mathrm{Ti}$ adatom mobility at temperatures $\leq 900 \mathrm{~K}$ is presently beyond the ability of AIMD/DFT simulations (see Table 2 and Fig. 6). The number of diffusion events recorded during AIMD runs is too low and produces uncertainties $\sigma_{\mathrm{D}}$ that exceed the magnitude of $\mathrm{D}_{\mathrm{s}}(\mathrm{T})$ values obtained from Eqs. (2) - (5). At $\mathrm{T} \geq 900 \mathrm{~K}$, AIMD $\mathrm{D}_{\mathrm{s}}$ values are defined within a reasonably small uncertainty range, and confirm the reliability of the CMD estimations. This lends confidence that CMD-based $\mathrm{D}_{\mathrm{s}}$ results are also reliable at $\mathrm{T}<900 \mathrm{~K}$. The uncertainties $\sigma_{\mathrm{D}}$ associated with the CMD results are relatively small considering that measured bulk TiN selfdiffusion coefficients values range over three to four orders of magnitude [14,16,17].

Ti adatom distribution probabilities $\mathbf{P}(\overrightarrow{\mathbf{r}}, \mathbf{t}, \mathbf{T})$ on $\mathrm{TiN}(001)$ are two-dimensional Gaussian functions provided that the time $t$ in Eqs. (2) - (5) is much larger than the inverse jump rate at a given temperature. Thus, a linear interpolation of $\ln \left[\mathrm{D}_{\mathrm{s}}(\mathrm{T})\right]$ vs. $1 / \mathrm{T}$ (Eq. (8)) yields values for activation energy and attempt frequency which correspond to ideal radially isotropic Ti adatom diffusion on TiN(001) terraces. From AIMD calculations, $\mathbf{E}_{\mathbf{a}}^{\prime}=(0.46 \pm 0.23) \mathrm{eV}$ and $\mathbf{A}^{\prime}=(3.5 \pm$ 2.8) $\times 10^{-4} \mathrm{~cm}^{2} \mathrm{~s}^{-1}$; for CMD $\mathbf{E}_{\mathbf{a}}^{\prime}=(0.60 \pm 0.02) \mathrm{eV}$ and $\mathbf{A}^{\prime}=(17.2 \pm 3.8) \times 10^{-4} \mathrm{~cm}^{2} \mathrm{~s}^{-1} . \mathrm{D}_{\mathrm{s}}(\mathrm{T})$ values are obtained from the relationship $D_{s}(T)=A^{\prime} \exp \left[-E_{a}^{\prime} /\left(k_{B} T\right)\right]$.

Determining diffusion coefficients from probability distribution functions, Eqs. (2) - (5), implicitly assumes that adatom jumps are completely uncorrelated. This, however, may not be the case. For example, when the rate of long jumps is significant (such as observed experimentally for $\mathrm{W}$ and $\mathrm{Pd}$ on $\mathrm{W}(110)$ and $\mathrm{W}(211)$ surfaces $[55,56])$, approximating atomic displacement distribution probabilities by counting a double-jump as two uncorrelated single jumps leads to an incorrect prediction of mass transport as a function of time. This occurs because a double-jump actually corresponds to two consecutive highly-correlated single jumps occurring along the same 
diffusion path. To probe the effect of correlated jumps on the Ti adatom diffusion coefficient, we calculate $\mathrm{D}_{\mathrm{s}}^{\mathrm{cor}}$ from Eq. (9) by directly tracking the adatom mean-square displacement on large TiN(001) simulation supercells during 10-ns CMD runs (see Section 2). Due to insufficient simulation time, correlated diffusion coefficients $\mathbf{D}_{\mathbf{s}}^{\text {corr }}$ do not exhibit a perfectly linear Arrhenius behavior (see Fig. 7). However, $\mathrm{D}_{\mathrm{s}}^{\mathrm{cor}}$ values are lower than uncorrelated $C M D \mathrm{D}_{\mathrm{s}}$ values at all temperatures, indicating that, because of correlation effects, the actual $\mathrm{Ti}$ adatom diffusion coefficients are smaller than those based purely on random walks. By linearly interpolating the $\ln ($ $\left.\mathrm{D}_{\mathrm{s}}^{\mathrm{cor}}(\mathrm{T})\right)$ data set vs. $1 / \mathrm{T}$, we extract effective jump activation energies and attempt frequencies $\mathbf{E}_{\mathrm{a}}^{\prime \text { corr }}=(0.55 \pm 0.10) \mathrm{eV}$ and $\mathrm{A}^{\prime \text { corr }}=(4.5 \pm 3.2) \times 10^{-4} \mathrm{~cm}^{2} \mathrm{~s}^{-1}$

Due to the short simulation times in AIMD, it is not possible to probe the effect of correlated adatom hops on diffusion coefficients. As noted at the beginning of this Section, because of the low frequency of double jumps in AIMD runs, each of these events is counted as two single jumps. This approximation inevitably yields underestimated AIMD $\mathrm{D}_{\mathrm{s}}$ values, as the atomicdisplacement Gaussian probability distribution generated by the random walk of an adspecies performing $\mathrm{N}$ hops, in which $\mathrm{N}$ is a large integer number, of length $\mathrm{L}$ is narrower than that obtained from stochastically oriented $\mathrm{N} / \mathrm{n}$ jumps ( $\mathrm{n}$ is an integer) of length $\mathrm{n} \times \mathrm{L}$. This error will be larger for higher $\mathrm{k}_{\text {double }} / \mathrm{k}_{\text {single }}$ jump-rate ratios. Thus, for $\mathrm{Ti}$ adatom diffusion coefficients obtained from AIMD adatom-probability distributions, the error introduced in $\mathrm{D}_{\mathrm{s}}$ by counting each double-jump as two single-jumps, partially cancels out the error caused by neglecting correlation effects between consecutive jumps. Accounting for the different limitations of the techniques used to determine surface diffusivity, the most reliable estimates of convolved diffusion barriers $\mathbf{E}_{\mathbf{a}}^{\prime}$ and jump attempt frequencies $\mathbf{A}^{\prime}$ for Ti adatom radially isotropic diffusion on $\mathrm{TiN}(001)$ are summarized in Table 3. Remarkably, AIMD $\mathrm{E}_{\mathrm{a}}^{\prime}$ and $\mathrm{A}^{\prime}$ values are close to $\mathrm{E}_{\mathrm{a}}^{\prime \text { corr }}$ and $\mathrm{A}^{\prime \text { corr }}$ values obtained from CMD. 
Using CMD-calculated $\mathrm{D}_{\mathrm{s}}$ and $\mathrm{D}_{\mathrm{s}}^{\mathrm{cor}}$ values in Eq. (7), we determine the dependence of the Ti adatom average displacement $\langle\alpha>$ on temperature $\mathrm{T}$ and time $\mathrm{t}$ (see Fig. 8). $\langle\alpha>$ obtained from CMD results are always within the uncertainty range of those obtained from AIMD.

\section{Conclusions}

AIMD/DFT and CMD/MEAM simulations reveal that Ti adatoms on TiN(001) reside in fourfold hollow positions, surrounded by two Ti and two $\mathrm{N}$ surface atoms, and that the preferred migration path between these sites is along in-plane $<100>$ channels. Activation energies $E_{a}$ and prefactors A for each of the three primary diffusion pathway are extracted from their jump-rate dependences on temperature. Uncorrelated jump probabilities $A \exp \left[-\mathrm{E}_{\mathrm{a}} /\left(\mathrm{k}_{\mathrm{B}} \mathrm{T}\right)\right]$ are used to determine diffusion coefficients $\mathrm{D}_{\mathrm{s}}$ which quantify the mobility of adatoms following purely random trajectories on the terrace. $\mathrm{AIMD} \mathrm{D}_{\mathrm{s}}$ results are consistent with $\mathrm{CMD}$, and thus validate the predictions of our MEAM parameterization. CMD simulations are employed to probe the effect of increasing supercell areas (phonon wavelength degrees of freedom) on adatom hop rates and $\mathrm{D}_{\mathrm{s}}$. Finally, by directly tracking adspecies mean-square displacements during CMD runs, we observe that actual adatom diffusivities $\mathrm{D}_{\mathrm{s}}^{\mathrm{cor}}(\mathrm{T})$, inherently accounting for correlation effects between consecutive jumps, are smaller than $\mathrm{D}_{\mathrm{s}}(\mathrm{T})$ at all temperatures.

\section{Acknowledgments}

Calculations were performed using the resources provided by the Swedish National Infrastructure for Computing (SNIC), on the Triolith Cluster located at the National Supercomputer Centre (NSC) in Linköping and on the Akka and Abisko clusters located at the High Performance Computing Center North (HPC2N) in Umeå, Sweden. We gratefully acknowledge financial support from the Knut and Alice Wallenberg Foundation (isotope project), the Swedish Research Council (VR) Linköping Linnaeus Initiative LiLi-NFM (grant 2008-6572), and the Swedish Government Strategic Research Area Grant in Materials Science on Advanced Functional Materials. 


\section{References}

[1] T. Reeswinkel, D.G. Sangiovanni, V. Chirita, L. Hultman, J.M. Schneider, Surface and Coatings Technology 205 (2011) 4821.

[2] H. Kindlund, D.G. Sangiovanni, J. Lu, J. Jensen, V. Chirita, I. Petrov, J.E. Greene, L. Hultman, Journal of Vacuum Science \& Technology A 32 (2014) 030603.

[3] H. Kindlund, D.G. Sangiovanni, L. Martínez-de-Olcoz, J. Lu, J. Jensen, J. Birch, I. Petrov, J.E. Greene, V. Chirita, L. Hultman, Applied Physics Letters Materials 1 (2013) 042104.

[4] L.E. Toth, Transition Metal Carbides and Nitrides, Academic Press, New York, 1971.

[5] J.M. Molarius, A.S. Korhonen, E. Harju, R. Lappalainen, Surface \& Coatings Technology 33 (1987) 117.

[6] V.R. Parameswaran, J.P. Immarigeon, D. Nagy, Surface \& Coatings Technology 52 (1992) 251.

[7] M.A. Nicolet, Thin Solid Films 52 (1978) 415-443.

[8] I. Petrov, E. Mojab, F. Adibi, J.E. Greene, L. Hultman, J.E. Sundgren, Journal of Vacuum Science \& Technology A 11 (1993) 11.

[9] J.S. Chun, I. Petrov, J.E. Greene, Journal of Applied Physics 86 (1999) 3633.

[10] J.S. Chun, P. Desjardins, I. Petrov, J.E. Greene, C. Lavoie, C. Cabral, Thin Solid Films 391 (2001) 69.

[11] J.S. Chun, P. Desjardins, C. Lavoie, C.S. Shin, C. Cabral, I. Petrov, J.E. Greene, Journal of Applied Physics 89 (2001) 7841.

[12] H. Matzke, Diffusion in carbides and nitrides, Kluwer Academic Publ., Dordrecht, 1990.

[13] H. Matzke, Philosophical Magazine 64 (1991) 1181.

[14] L. Hultman, Vacuum 57 (2000) 1.

[15] J.E. Sundgren, Thin Solid Films 128 (1985) 21.

[16] F.W. Wood, O.G. Paasche, Thin Solid Films 40 (1977) 131.

[17] F. Anglezioabautret, B. Pellissier, M. Miloche, P. Eveno, Journal of the European Ceramic Society, 8, (1991) 299.

[18] S. Kodambaka, V. Petrova, A. Vailionis, P. Desjardins, D.G. Cahill, I. Petrov, J.E. Greene, Surface Review and Letters 7 (2000) 589.

[19] S. Kodambaka, V. Petrova, A. Vailionis, P. Desjardins, D.G. Cahill, I. Petrov, J.E. Greene, Thin Solid Films 392 (2001) 164.

[20] S. Kodambaka, V. Petrova, A. Vailionis, I. Petrov, J.E. Greene, Surface Science 526 (2003) 85. 
[21] F. Watanabe, S. Kodambaka, W. Swiech, J.E. Greene, D.G. Cahill, Surface Science 572 (2004) 425.

[22] S. Kodambaka, N. Israeli, J. Bareno, W. Swiech, K. Ohmori, I. Petrov, J.E. Greene, Surface Science 560 (2004) 53.

[23] J. Bareno, S. Kodambaka, S.V. Khare, W. Swiech, V. Petrova, I. Petrov, J.E. Greene, TiN surface dynamics: Role of surface and bulk mass transport processes, Mexico City, Mexico, (2006) 205.

[24] D. Gall, S. Kodambaka, M.A. Wall, I. Petrov, J.E. Greene, Journal of Applied Physics 93 (2003) 9086.

[25] B. Alling, P. Steneteg, C. Tholander, F. Tasnadi, I. Petrov, J.E. Greene, L. Hultman, Physical Review B 85 (2012) 245422.

[26] Y. Ren, X.J. Liu, X. Tan, E. Westkamper, Computational Materials Science 77 (2013) 102.

[27] G. Henkelman, B.P. Uberuaga, H. Jonsson, Journal of Chemical Physics 113 (2000) 9901.

[28] G. Henkelman, H. Jonsson, Journal of Chemical Physics 113 (2000) 9978.

[29] D.G. Truhlar, B.C. Garrett, S.J. Klippenstein, Journal of Physical Chemistry 100 (1996) 12771.

[30] C. Wert, C. Zener, Physical Review 76 (1949) 1169.

[31] G.H. Vineyard, J. Phys. Chem. Solids 3 (1957) 121.

[32] E.I. Isaev, S.I. Simak, I.A. Abrikosov, R. Ahuja, Y.K. Vekilov, M.I. Katsnelson, A.I. Lichtenstein, B. Johansson, Journal of Applied Physics 101 (2007) 123519.

[33] R. Car, M. Parrinello, Physical Review Letters 55 (1985) 2471.

[34] A.V. Ruban, I.A. Abrikosov, Reports on Progress in Physics 71 (2008) 046501.

[35] L. Schimka, J. Harl, A. Stroppa, A. Gruneis, M. Marsman, F. Mittendorfer, G. Kresse, Nature Materials 9 (2010) 741.

[36] P.J. Feibelman, B. Hammer, J.K. Norskov, F. Wagner, M. Scheffler, R. Stumpf, R. Watwe, J. Dumesic, Journal of Physical Chemistry B 105 (2001) 4018.

[37] D.G. Sangiovanni, D. Edström, L. Hultman, V. Chirita, I. Petrov, J.E. Greene, Physical Review B 86 (2012) 155443.

[38] D. Edström, D.G. Sangiovanni, L. Hultman, V. Chirita, I. Petrov, J.E. Greene, Thin Solid Films (in press) (2014) http://dx.doi.org/10.1016/j.tsf.2014.02.053.

[39] D.G. Sangiovanni, D. Edström, L. Hultman, I. Petrov, J.E. Greene, V. Chirita, Surface Science 624 (2014) 25.

[40] H. Ljungcrantz, M. Oden, L. Hultman, J.E. Greene, J.E. Sundgren, Journal of Applied Physics 80 (1996) 6725. 
[41] J.E. Greene, J.E. Sundgren, L. Hultman, I. Petrov, D.B. Bergstrom, Applied Physics Letters 67 (1995) 2928.

[42] M.P. Allen, D.J. Tildesley, Computer simulation of liquids, Oxford University Press, 1989.

[43] B.J. Lee, M.I. Baskes, Physical Review B 62 (2000) 8564.

[44] S. Plimpton, Journal of Computational Physics 117 (1995) 1.

[45] In our CMD simulations, we use a LAMMPS ialloy parameter value $=0$, and ibar parameter value = 3. In Ref. [Y. M. Kim, B. J. Lee, Acta Mater. 56 (2008) 3481], ialloy =2, ibar = 3.

[46] M.A. Wall, D.G. Cahill, I. Petrov, D. Gall, J.E. Greene, Surface Science 581 (2005) L122.

[47] G. Kresse, J. Hafner, Physical Review B 47 (1993) 558.

[48] J.P. Perdew, K. Burke, Y. Wang, Physical Review B 54 (1996) 16533.

[49] P.E. Blöchl, Physical Review B 50 (1994) 17953.

[50] D.G. Sangiovanni, V. Chirita, L. Hultman, Physical Review B 81 (2010) 104107.

[51] H. Brune, K. Bromann, H. Roder, K. Kern, J. Jacobsen, P. Stoltze, K. Jacobsen, J. Norskov, Physical Review B 52 (1995) 14380.

[52] M. Marlo, V. Milman, Physical Review B 62 (2000) 2899.

[53] T. Nagy, T. Turanyi, International Journal of Chemical Kinetics 43 (2011) 359.

[54] L. Tsetseris, N. Kalfagiannis, S. Logothetidis, S.T. Pantelides, Physical Review Letters 99 (2007) 4.

[55] D.C. Senft, G. Ehrlich, Physical Review Letters 74 (1995) 294.

[56] G. Antczak, G. Ehrlich, Physical Review Letters 92 (2004) 166105. 


\section{Tables}

\begin{tabular}{|l|c|c|c|c|}
\hline \multirow{2}{*}{ Ti/TiN $(001)$} & \multicolumn{2}{|c|}{ AIMD } & \multicolumn{2}{c|}{ CMD } \\
\cline { 2 - 5 } & $\mathrm{E}_{\mathrm{a}}(\mathrm{eV})$ & $\mathrm{A}\left(10^{13} \mathrm{~s}^{-1}\right)$ & $\mathrm{E}_{\mathrm{a}}(\mathrm{eV})$ & $\mathrm{A}\left(10^{13} \mathrm{~s}^{-1}\right)$ \\
\hline All jumps & $0.51 \pm 0.03$ & $0.79 \pm 0.14$ & $0.62 \pm 0.01$ & $2.76 \pm 0.23$ \\
Single $<100>$ & $0.54 \pm 0.06$ & $0.53 \pm 0.22$ & $0.57 \pm 0.01$ & $1.09 \pm 0.10$ \\
Single $<110>$ & $0.44 \pm 0.07$ & $0.15 \pm 0.08$ & $0.81 \pm 0.03$ & $0.51 \pm 0.14$ \\
Double $<100>$ & - & - & $0.84 \pm 0.01$ & $3.36 \pm 0.15$ \\
\hline
\end{tabular}

Table 1. AIMD and CMD activation energies $E_{a}$ and prefactors $A$, calculated using Eq. (1), for preferred $\mathrm{Ti}$ adatom diffusion pathways on $\mathrm{TiN}(001)$.

\begin{tabular}{|c|c|c|c|}
\hline \multirow[b]{3}{*}{$\mathrm{T}(\mathrm{K})$} & \multicolumn{3}{|c|}{$\mathrm{D}_{\mathrm{s}}\left(\mathrm{cm}^{2} \mathrm{~s}^{-1}\right)$} \\
\hline & \multirow{2}{*}{$\begin{array}{c}\text { Correlated } \\
\text { CMD }\end{array}$} & \multicolumn{2}{|c|}{ Uncorrelated } \\
\hline & & CMD & AIMD \\
\hline 300 & - & $(1.3 \pm 0.6) \times 10^{-13}$ & $\sigma_{\mathrm{D}}>\mathrm{D}$ \\
\hline 600 & $(1.0 \pm 0.7) \times 10^{-8}$ & $(1.5 \pm 0.2) \times 10^{-8}$ & $\sigma_{\mathrm{D}}>\mathrm{D}$ \\
\hline 900 & $(5.0 \pm 2.1) \times 10^{-7}$ & $(7.3 \pm 0.9) \times 10^{-7}$ & $(9.2 \pm 8.9) \times 10^{-7}$ \\
\hline 1000 & ${ }^{*}(5.2 \pm 1.9) \times 10^{-7}$ & $(1.6 \pm 0.2) \times 10^{-6}$ & $(1.7 \pm 1.5) \times 10^{-6}$ \\
\hline 1200 & $(3.9 \pm 1.4) \times 10^{-6}$ & $(5.1 \pm 0.6) \times 10^{-6}$ & $(4.1 \pm 3.2) \times 10^{-6}$ \\
\hline 1500 & $(0.5 \pm 0.2) \times 10^{-5}$ & $(1.6 \pm 0.3) \times 10^{-5}$ & $(1.0 \pm 0.7) \times 10^{-5}$ \\
\hline 1800 & - & $(3.6 \pm 0.5) \times 10^{-5}$ & $(1.8 \pm 1.2) \times 10^{-5}$ \\
\hline
\end{tabular}

${ }^{*}$ From reference [37].

Table 2. Ti/TiN(001) adatom diffusion coefficient $D_{s}(T)$ obtained from CMD and AIMD simulations as a function of temperature $\mathrm{T}$ for correlated and uncorrelated events.

\begin{tabular}{|l|c|c|c|c|c|c|}
\hline \multirow{2}{*}{} & \multicolumn{2}{|c|}{ Correlated } & \multicolumn{4}{c|}{ Uncorrelated } \\
\cline { 2 - 7 } & \multicolumn{2}{|c|}{ CMD } & \multicolumn{2}{c|}{ CMD } & \multicolumn{2}{c|}{ AIMD } \\
\cline { 2 - 7 } & $\begin{array}{c}\mathrm{E}_{\mathbf{a}}^{\prime \text { corr }} \\
(\mathrm{eV})\end{array}$ & $\begin{array}{c}\mathrm{A}^{\prime \text { corr }} \\
\left(10^{-4} \mathrm{~cm}^{2} \mathrm{~s}^{-1}\right)\end{array}$ & $\begin{array}{c}\mathrm{E}_{\mathrm{a}}^{\prime} \\
(\mathrm{eV})\end{array}$ & $\begin{array}{c}\mathrm{A}^{\prime} \\
\left(10^{-4} \mathrm{~cm}^{2} \mathrm{~s}^{-}\right. \\
1\end{array}$ & $\begin{array}{c}\mathrm{E}_{\mathrm{a}}^{\prime} \\
(\mathrm{eV})\end{array}$ & $\begin{array}{c}\mathrm{A}^{\prime} \\
\left(10^{-4} \mathrm{~cm}^{2} \mathrm{~s}^{-1}\right)\end{array}$ \\
\hline $\begin{array}{l}\text { Radially } \\
\text { isotropic } \\
\text { diffusion }\end{array}$ & $\begin{array}{c}0.55 \pm \\
0.10\end{array}$ & $4.5 \pm 3.2$ & $0.60 \pm 0.02$ & $17.2 \pm 3.8$ & $\begin{array}{c}0.46 \pm \\
0.23\end{array}$ & $3.5 \pm 2.8$ \\
\hline
\end{tabular}

Table 3. AIMD and CMD convolved activation energy $\mathbf{E}_{\mathbf{a}}^{\prime}$ and attempt frequency $\mathbf{A}^{\prime}$ corresponding to $\mathrm{Ti}$ adatom ideal isotropic diffusion on $\mathrm{TiN}(001)$ as determined by AIMD and CMD. 


\section{Figures}

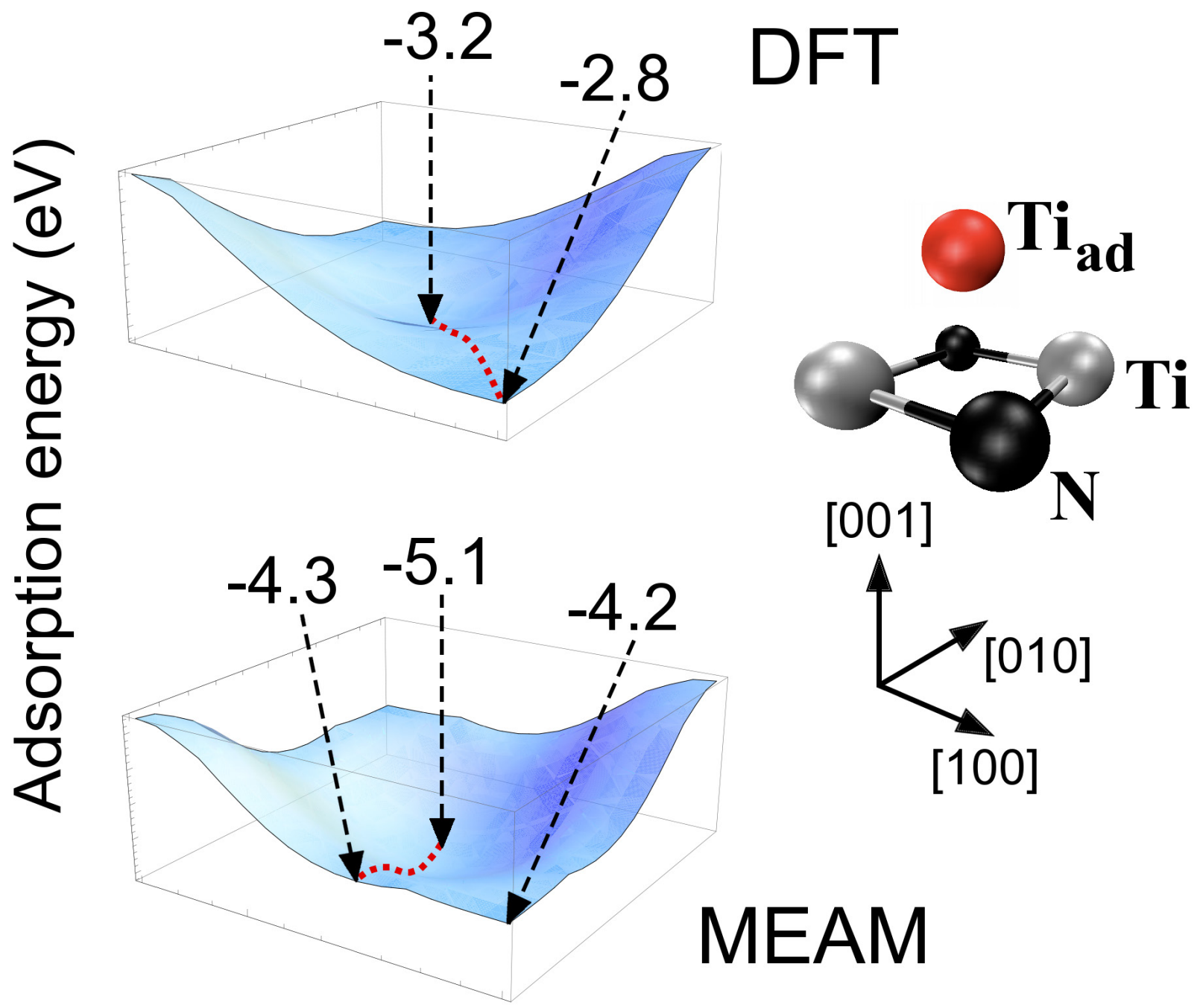

Figure 1. Adsorption energy landscapes for Ti adatoms on a TiN(001) surface unit cell. A schematic view of the surface unit cell is shown to the right of the image. Adsorption energies are calculated with respect to the total energy of a $\mathrm{Ti}$ atom in the gas phase. Minimum energy paths connecting stable fourfold hollow sites, determined from the curvature of the potential energy surface, and confirmed by NEB calculations, are shown by the red dotted lines. Adsorption energies are indicated only for the most stable adsorption site (fourfold hollow), the metastable epitaxial site, and, in MEAM results, for the energetically-favored position at the surface unit cell boundary, a Ti$\mathrm{N}$ bridge site closer to the $\mathrm{N}$ surface atom. 

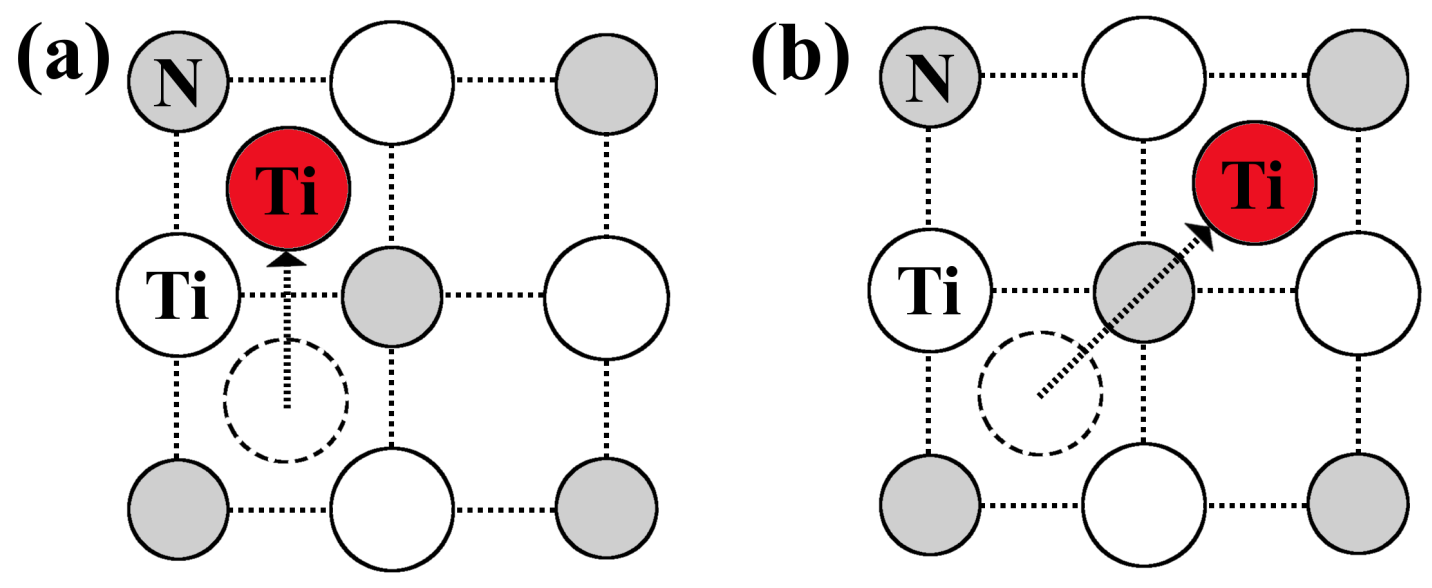

Figure 2. Schematic representation of $\mathrm{Ti}$ adatom net translations on $\mathrm{TiN}(001)$ surfaces connecting stable fourfold hollow site positions as observed in AIMD and CMD videos. (a) In-plane $<100>$ single jumps and (b) in-plane $<110>$ single jumps crossing metastable epitaxial sites atop $\mathrm{N}$ surface atoms. 


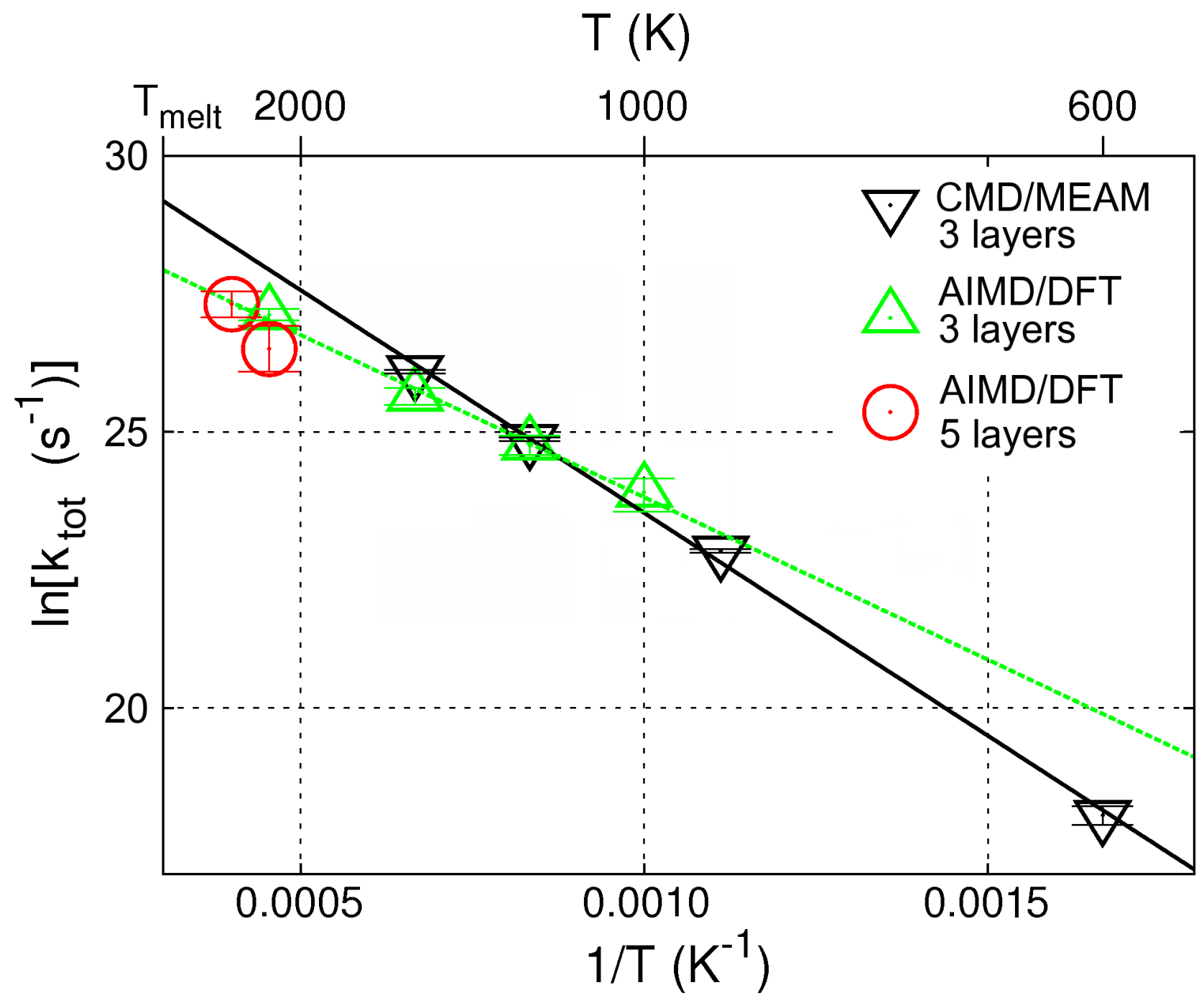

Figure 3. Ti adatom total jump rate $\mathrm{k}_{\mathrm{tot}}$, obtained from AIMD and CMD simulations, vs. inverse temperature $1 / \mathrm{T} . \ln \left(\mathrm{k}_{\mathrm{tot}}\right)$ values obtained from AIMD runs on thicker simulation slabs comprised of five $\operatorname{TiN}(001)$ atomic layers is within the uncertainty range bars indicate uncertainties tayeroAtM地 results. Error 

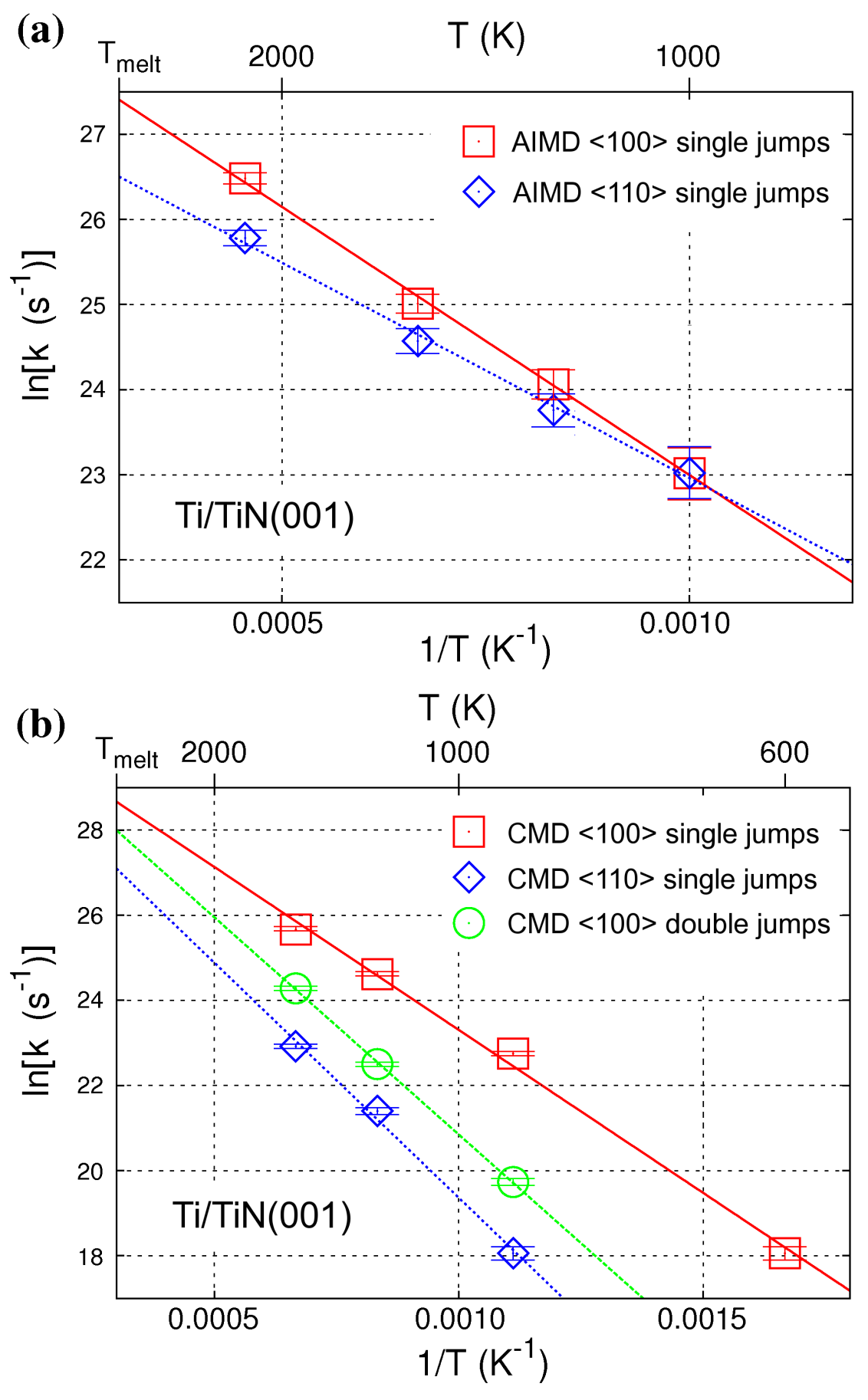

Figure 4. Ti adatom jump rate $\ln (\mathrm{k})$ vs. inverse temperature $1 / \mathrm{T}$ for the primary diffusion pathways on TiN(001). (a) AIMD simulation results and (b) CMD simulation results. Error bars indicate uncertainties $\square$ (see Section 2). 


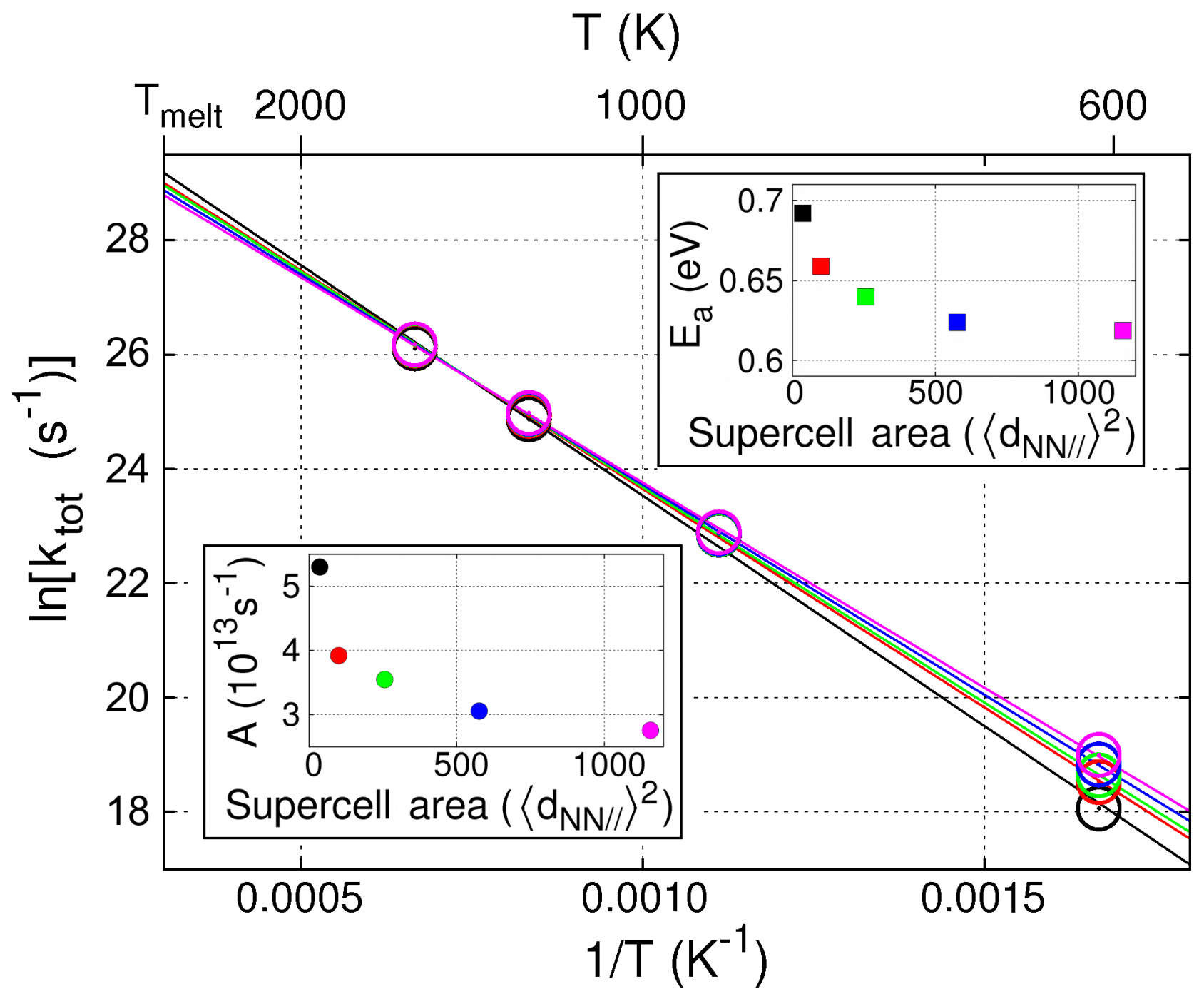

Figure 5. Ti adatom total jump rate $\mathrm{k}_{\text {tot}}$, obtained from CMD simulations, vs. inverse temperature $1 / \mathrm{T}$. The $\mathrm{TiN}(001)$ supercell area is increased from $3 \times 3$ (black) to $5 \times 5$ (red), $8 \times 8$ (green), $12 \times 12$ (blue), and 17x17 (purple) surface unit cells. The inserts show diffusion activation energy and attempt frequency dependence on the $\mathrm{TiN}(001)$ supercell area. 


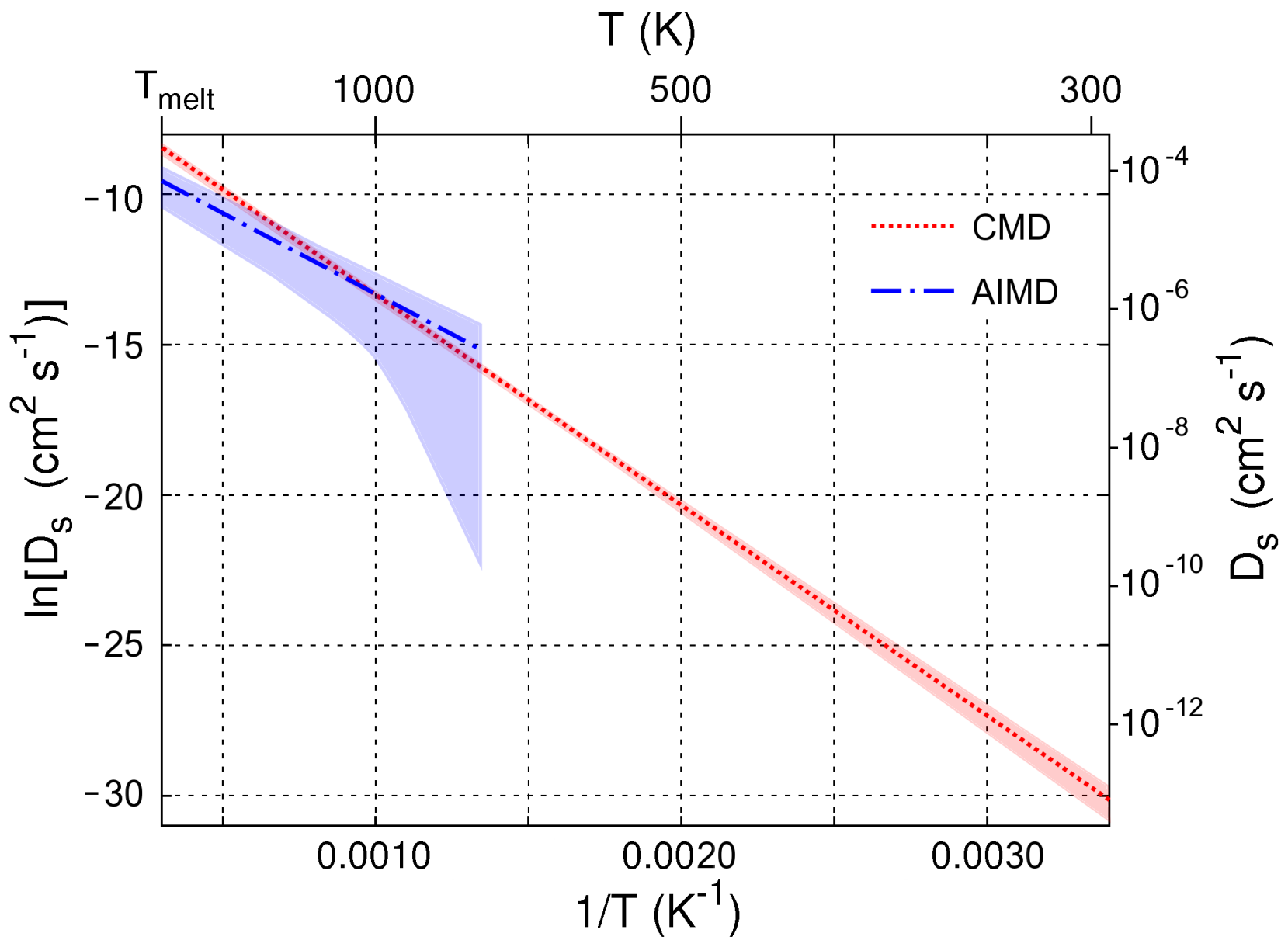

Figure 6. $C M D$ and $A I M D T i$ adatom diffusion coefficient $D_{s}$ vs. inverse temperature $1 / T$ for uncorrelated transition probabilities. Shaded regions correspond to uncertainties $\sigma_{\square}$ (see Section 2 and Table 2). 


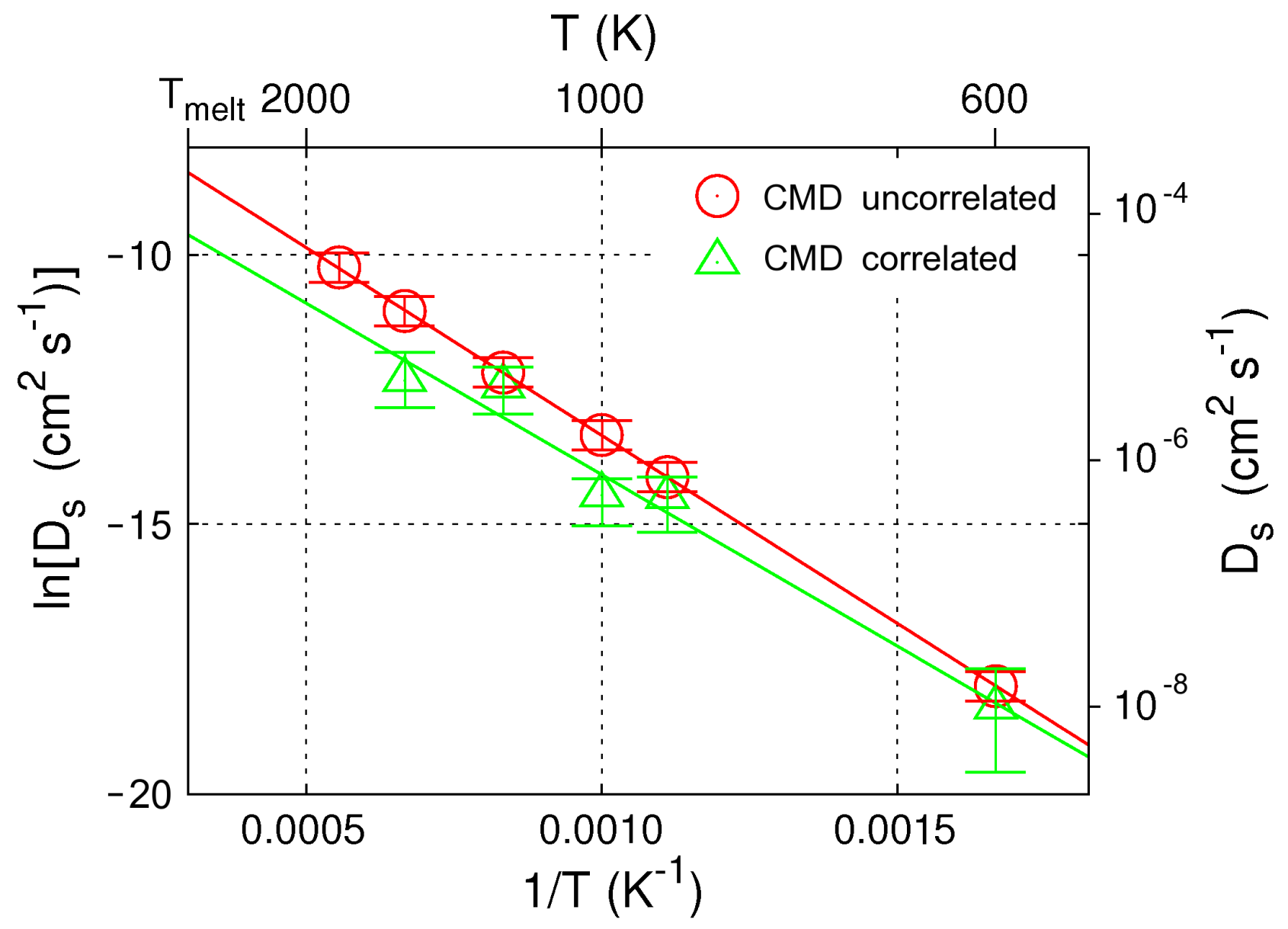

Figure 7. Ti adatom diffusion coefficient $D_{s}$ vs. inverse temperature $1 / T$ obtained from CMD results. Open red circles (green triangles) correspond to diffusion coefficients determined from uncorrelated (correlated) Ti adatom transition probabilities between stable sites on TiN(001). Error bars indicate uncertainties $\sigma_{\square}($ see Section 2). 


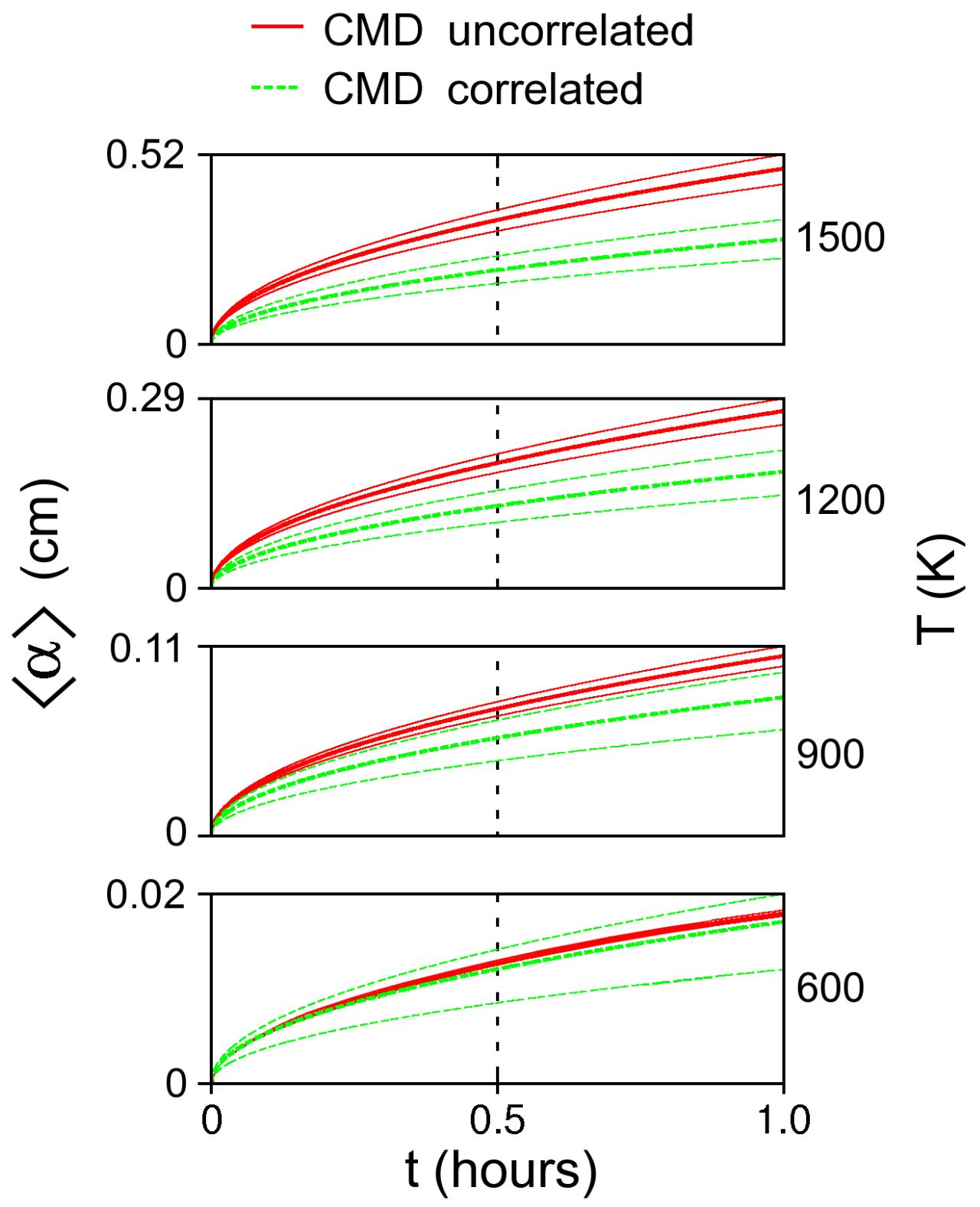

Figure 8. Ti adatom average displacement $\langle\alpha\rangle$ (thick lines), obtained from Eq. (7) parameterized with CMD diffusion coefficients deriving from correlated (green dashed lines) and uncorrelated (solid red lines) jumps, as a function of temperature $\mathrm{T}$ and time $\mathrm{t}$. Thin lines define uncertainty ranges $\left[D_{s}(T) \pm \sigma_{D}(T)\right]$. 